\title{
Common Fixed Point Theorems in Random Probabilistic Metric Spaces
}

\author{
Rajesh Shrivastava $\&$ Richa Gupta ${ }^{2}$ \\ 1. Prof. \& Head, Department of Mathematics, Govt. Science \& Commerce college Benazir Bhopal,India \\ 2. Head, Department of Mathematics, RKDF institute of Science \& Technology Bhopal, India
}

Abstract: We prove some Common Fixed Point theorems for Random Operator in Probabilistic metric spaces, by using some new type of contractive conditions taking self mappings.

Key Words: - Probabilistic metric spaces, Random Operator, Random Fixed Point, Measurable Mapping, AMS Subject Classification: - 47H10, 54H25.

\section{Introduction}

In 1942, Menger [5] was first who thought about distance distribution function in metric space and introduced the concept of probabilistic metric space. He replaced distance function $d(x, y)$, the distance between two point x,y by distance distribution function $F x, y(t)$ where the value of $F x, y(t)$ is interpreted as probability that the distance between $x, y$ is less than $t, t>0$. The study of fixed point theorem in probabilistic metrics space is useful in the study of existence of solution of operator equation in probabilistic metric space probabilistic functional analysis.

PM space has a nice topological properties. Many different topological structures may be defined on a PM space. The one That has received the most attention to date is the strong topology and it is the principle tool of this study. The convergence with respect to this topology is called strong convergence.

Schweizer and Sklar [1], developed the study of fixed point theory in probabilistic metric spaces. In 1966, Sehgal [12] initiated the study of contraction mapping theorem in probabilistic metric spaces. Several interesting and elegant result have been proved by various author in probabilistic metric spaces.

In 2005, Mihet [2] proved a fixed point theorem concerning probabilistic contractions satisfying an implicit relation. The purpose of the present paper is to prove a common fixed point theorem for six mappings via pointwise R-weakly commuting mappings in probabilistic metric spaces satisfying contractive type implicit relations. This generalizes several known results in the literature including those of Kumar and Pant [8], Kumar and Chugh [7] and others.

Definition 2.1.1: Let $R$ denote the set of reals and $R^{+}$the non-negative reals. A mapping $F: R \rightarrow R^{+}$is called a distribution function if it is non decreasing left continuous with $\inf _{t \in R} F(t)=0$ and $\sup _{t \in R} F(t)=1$

Definition 2.1.2: A probabilistic metric space is an ordered pair (X,F) where $X$ is a nonempty set, $L$ be set of all distribution function and $F: X \times X \rightarrow L$. We shall denote the distribution function by $F(p, q)$ or $F_{p, q} ; p, q \in X$ and $F_{p, q}(x)$ will represents the value of $F(p, q)$ at $x \in R$. The function $F(p, q)$ is assumed to satisfy the following conditions:

2.1.2(a) $\mathrm{F}_{\mathrm{p}, \mathrm{q}}(\mathrm{x})=1$ for all $\mathrm{x}>0$ if and only if $p=q$

2.1.2(b) $\mathrm{F}_{\mathrm{p}, \mathrm{q}}(0)=0$ for every $\mathrm{p}, \mathrm{q} \in \mathrm{X}$

2.1.2(c) $\mathrm{F}_{\mathrm{p}, \mathrm{q}}=\mathrm{F}_{\mathrm{q}, \mathrm{p}}$ for every $\mathrm{p}, \mathrm{q} \in \mathrm{X}$

2.1.2(d) $\mathrm{F}_{\mathrm{p}, \mathrm{q}}(\mathrm{x})=1$ and $\mathrm{F}_{\mathrm{q}, \mathrm{r}}(\mathrm{y})=1$ then $\mathrm{F}_{\mathrm{p}, \mathrm{r}}(\mathrm{x}+\mathrm{y})=1$

for every $p, q, r \in X$.

In metric space $(X, d)$, the metric $d$ induces a mapping $F: X \times X \rightarrow L$ such that $F_{p, q}(x)=F_{p, q}=$

$H(x-d(p, q))$ for every $p, q \in X$ and $x \in R$, where $H$ is the distribution function defined as

$$
H(x)=\left\{\begin{array}{l}
0, \text { if } x \leq 0 \\
1, \text { if } x>0
\end{array}\right.
$$

Definition 2.1.3: A mapping $\Delta:[0,1] \times[0,1] \rightarrow[0,1]$ is called t-norm if

2.1.3(a) $\Delta(\mathrm{a}, 1)=\mathrm{a} \forall \mathrm{a} \in[0,1]$

2.1.3(b) $\Delta(0,0)=0$, 


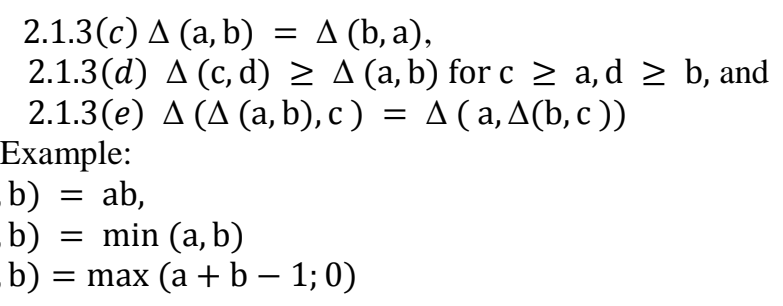

Definition 2.1.4: A Menger space is a triplet $(\mathrm{X}, \mathrm{F}, \Delta)$ where $(\mathrm{X}, \mathrm{F})$ a PM-space and $\Delta$ is is a t-norm with the following condition

$\mathrm{F}_{\mathrm{u}, \mathrm{w}}(\mathrm{x}+\mathrm{y}) \geq \Delta\left(\mathrm{F}_{\mathrm{u}, \mathrm{v}}(\mathrm{x}), \mathrm{F}_{\mathrm{v}, \mathrm{w}}(\mathrm{y})\right.$

The above inequality is called Menger's triangle inequality.

Definition 2.1.5: Let $(X, F, \Delta)$ be a Menger space. If $u \in X, \varepsilon>0, \lambda \in(0,1)$, then an $(\varepsilon, \lambda)$ neighbourhood of $\mathrm{u}$, denoted by $\mathrm{U}_{\mathrm{u}}(\varepsilon, \lambda)$ is defined as

$$
\mathrm{U}_{\mathrm{u}}(\varepsilon, \lambda)=\left\{\mathrm{v} \in \mathrm{X} ; \mathrm{F}_{\mathrm{u}, \mathrm{v}}(\varepsilon)>1-\lambda\right\} .
$$

If $(X, F, \Delta)$ be a Menger space with the continuous t-norm t, then the family $U_{u}(\varepsilon, \lambda) ; u \in X ; \varepsilon>0, \lambda \in$ $(0,1)$ of neighbourhood induces a hausdorff topology on $X$ and if $\sup _{a<1} \Delta(a, a)=1$, it is metrizable.

Definition 2.1.6: A sequence $\left\{p_{n}\right\}$ in $(X, F, \Delta)$ is said to be convergent to a point $p \in X$ if for every $\varepsilon>0$ and $\lambda>0$, there exists an integer $N=N(\varepsilon, \lambda)$ such that $p_{n} \in U_{p}(\varepsilon, \lambda)$ for all $n \geq N$ or equivalently $F_{x_{n}, x}(\epsilon)>$ $1-\lambda$ for all $\mathrm{n} \geq \mathrm{N}$.

Definition 2.1.7: A sequence $\left\{\mathrm{p}_{\mathrm{n}}\right\}$ in $(\mathrm{X}, \mathrm{F}, \Delta)$ is said to be Cauchy sequence if for every $\varepsilon>0$ and $\lambda>0$, there exists an integer $N=N(\varepsilon, \lambda)$ such that $F_{p_{n}, p_{m}}(\epsilon)>1-\lambda$ for all $n, m \geq N$.

Definition 2.1.8: A Menger space $(\mathrm{X}, \mathrm{F}, \Delta)$ with the continuous t-norm $\Delta$ is said to be complete if every Cauchy sequence in $\mathrm{X}$ converges to a point in $\mathrm{X}$.

Definition 2.1.9: A coincidence point (or simply coincidence) of two mappings is a point in their domain having the same image point under both mappings.

Formally, given two mappings $\mathrm{f}, \mathrm{g}: \mathrm{X} \rightarrow \mathrm{Y}$ we say that a point $\mathrm{X}$ in $\mathrm{X}$ is a coincidence point of $\mathrm{f}$ and $\mathrm{g}$ if $\mathrm{f}(\mathrm{x})=\mathrm{g}(\mathrm{x})$

Definition 2.1.10: Let $(\mathrm{X}, \mathrm{F}, \Delta)$ be a Menger space. Two mappings $\mathrm{f}, \mathrm{g}: \mathrm{X} \rightarrow \mathrm{X}$ are said to be weakly compatible if they commute at the coincidence point, i.e., the pair $\{\mathrm{f}, \mathrm{g}\}$ is weakly compatible pair if and only if $\mathrm{fx}=\mathrm{gx}$ implies that $\mathrm{fgx}=\mathrm{gfx}$.

Lemma 2.1.11: Let $\left\{p_{n}\right\}$ be a sequence in Menger space $(X, F, \Delta)$ where $\Delta$ is continuous and $\Delta(x, x) \geq x$ for all $\mathrm{x} \in[0,1]$. If there exists a constant $\mathrm{k} \in(0,1)$ such that $\mathrm{x}>0$ and $\mathrm{n} \in \mathbf{N} \quad \mathrm{F}_{\mathrm{p}_{\mathrm{n}}, \mathrm{p}_{\mathrm{n}+1}}(\mathrm{kx}) \geq \mathrm{F}_{\mathrm{p}_{\mathrm{n}-1}, \mathrm{p}_{\mathrm{n}}}(\mathrm{x})$, then $\left\{p_{n}\right\}$ is a Cauchy sequence.

Lemma 2.1.12: If $(X, d)$ is a metric space, then the metric $d$ induces a mapping $F: X \times X \rightarrow L$, defined by $F(p, q)=H(x-d(p, q)), p, q \in X$ and $x \in R$. Further more if $\Delta:[0,1] \times[0,1] \rightarrow[0,1]$ is defined by $\Delta(\mathrm{a}, \mathrm{b})=\min (\mathrm{a}, \mathrm{b})$, then $(\mathrm{X}, \mathrm{F}, \Delta)$ is a Menger space. It is complete if $(\mathrm{X}, \mathrm{d})$ is complete. The space $(\mathrm{X}, \mathrm{F}, \Delta)$ so obtained is called the induced Menger space.

Lemma2.1.13: Let $(X, F, \Delta)$ be a Menger space. ]. If there exists a constant $k \in(0,1)$ such that $F_{x, y}(k t) \geq$ $F_{x, y}(t)$, for all $x, y \in X$ and $t>0$ then $x=y$.

Now we give some definition which are used in this chapter.

Throughout this chapter, $(\Omega, \Sigma)$ denotes a measurable space. $\xi: \Omega \rightarrow X$ is a measurable selector. $\mathrm{X}$ is any non empty set.

Definition2.1.14: A random probabilistic metric space is an ordered pair $(\mathrm{X}, \mathrm{F}, \Omega)$ where $\mathrm{X}$ is a nonempty set, $\mathrm{L}$ be set of all distribution function and $\mathrm{F}: \mathrm{X} \times \mathrm{X} \rightarrow \mathrm{L}$. We shall denote the distribution function by $\mathrm{F}(\xi \mathrm{p}, \xi \mathrm{q})$ or $\mathrm{F}_{\xi \mathrm{p}, \xi q} ; \xi \mathrm{p}, \xi \mathrm{q} \in \mathrm{X}$ and $\mathrm{F}_{\xi \mathrm{p}, \xi q}(\mathrm{x})$ will represents the value of $\mathrm{F}(\xi \mathrm{p}, \xi \mathrm{q})$ at $\mathrm{x} \in \mathrm{R}$. The function $\mathrm{F}(\xi \mathrm{p}, \xi \mathrm{q})$ is assumed to satisfy the following conditions:

2.1.14(a) $\mathrm{F}_{\xi \mathrm{p}, \xi \mathrm{q}}(\mathrm{x})=1$ for all $\mathrm{x}>0$ if and only if $\xi \mathrm{p}=\xi \mathrm{q}$ 
2.1.14(b) $\mathrm{F}_{\xi \mathrm{p}, \xi q}(0)=0$ for every $\xi \mathrm{p}, \xi \mathrm{q} \in \mathrm{X}$

2.1.14(c) $\mathrm{F}_{\xi \mathrm{p}, \xi \mathrm{q}}=\mathrm{F}_{\xi \mathrm{q}, \xi \mathrm{p}}$ for every $\xi \mathrm{p}, \xi \mathrm{q} \in \mathrm{X}$

2.1.14(d) $\mathrm{F}_{\xi p, \xi q}(\mathrm{x})=1$ and $\mathrm{F}_{\xi q, \xi \mathrm{r}}(\mathrm{y})=1$ then $\mathrm{F}_{\xi \mathrm{p}, \xi_{\mathrm{r}}}(\mathrm{x}+\mathrm{y})=1$

for every $\xi \mathrm{p}, \xi \mathrm{q}, \xi \mathrm{r} \in \mathrm{X}$.

Definition 2.1.15: A Random Menger space is a triplet $(\mathrm{X}, \mathrm{F}, \Omega, \Delta)$ where $(\mathrm{X}, \mathrm{F}, \Omega)$ a RPM-space and $\Delta$ is is a tnorm with the following condition

$\mathrm{F}_{\xi \mathrm{u}, \xi \mathrm{w}}(\mathrm{x}+\mathrm{y}) \geq \Delta\left(\mathrm{F}_{\xi \mathrm{u}, \xi \mathrm{v}}(\mathrm{x}), \mathrm{F}_{\xi \mathrm{v}, \xi \mathrm{w}}(\mathrm{y})\right.$

The above inequality is called Menger's triangle inequality.

Definition 2.1.16: Let $(\mathrm{X}, \mathrm{F}, \Omega, \Delta)$ be a random menger space. If $\xi \mathrm{u} \in \mathrm{X}, \varepsilon>0, \lambda \in(0,1)$, then an $(\varepsilon, \lambda)$ neighbourhood of $\mathrm{u}$, denoted by $\mathrm{U}_{\xi \mathrm{u}}(\varepsilon, \lambda)$ is defined as

$$
\mathrm{U}_{\xi \mathrm{u}}(\varepsilon, \lambda)=\left\{\xi \mathrm{v} \in \mathrm{X} ; \mathrm{F}_{\xi \mathrm{u}, \xi \mathrm{v}}(\varepsilon)>1-\lambda\right\} \text {. }
$$

If $(\mathrm{X}, \mathrm{F}, \Omega, \Delta)$ be a random menger space with the continuous t-norm t, then the family $\mathrm{U}_{\mathrm{u}}(\varepsilon, \lambda), \xi \mathrm{u} \in$ $\mathrm{X}, \varepsilon>0, \lambda \in(0,1)$ of neighbourhood induces a hausdorff topology on $\mathrm{X}$ and if $\sup _{\mathrm{a}<1} \Delta(\mathrm{a}, \mathrm{a})=1$, it is metrizable.

Definition 2.1.17: A sequence $\left\{\xi \mathrm{p}_{\mathrm{n}}\right\}$ in $(\mathrm{X}, \mathrm{F}, \Omega, \Delta)$ is said to be convergent to a point $\xi \mathrm{p} \in \mathrm{X}$ if for every $\varepsilon>0$ and $\lambda>0$, there exists an integer $\mathrm{N}=\mathrm{N}(\varepsilon, \lambda)$ such that $\xi \mathrm{p}_{\mathrm{n}} \in \mathrm{U}_{\mathrm{p}}(\varepsilon, \lambda)$ for all $\mathrm{n} \geq \mathrm{N}$ or equivalently $\mathrm{F}_{\xi \mathrm{x}_{n}, \xi \mathrm{g}}(\epsilon)>1-\lambda$ for all $\mathrm{n} \geq \mathrm{N}$.

Definition 2.1.18: A sequence $\left\{\xi \mathrm{p}_{\mathrm{n}}\right\}$ in $(\mathrm{X}, \mathrm{F}, \Delta)$ is said to be Cauchy sequence if for every $\varepsilon>0$ and $\lambda>0$, there exists an integer $\mathrm{N}=\mathrm{N}(\varepsilon, \lambda)$ such that $\mathrm{F}_{\xi \mathrm{p}_{\mathrm{n}}, \xi \mathrm{p}_{\mathrm{m}}}(\epsilon)>1-\lambda$ for all $\mathrm{n}, \mathrm{m} \geq \mathrm{N}$.

Definition 2.1.19: A random menger space $(X, F, \Omega, \Delta)$ with the continuous t-norm $\Delta$ is said to be complete if every Cauchy sequence in $\mathrm{X}$ converges to a point in $\mathrm{X}$.

Definition 2.1.20: A coincidence point of two mappings is a point in their domain having the same image point under both mappings.

Formally, given two mappings $\mathrm{f}, \mathrm{g}: \mathrm{X} \rightarrow \mathrm{Y}$ we say that a point $\mathrm{x}$ in $\mathrm{X}$ is a coincidence point of $\mathrm{f}$ and $\mathrm{g}$ if $f(\xi x)=g(\xi x)$.

Definition 2.1.21: Let $(X, F, \Omega, \Delta)$ be a Menger space. Two mappings $f, g: X \rightarrow X$ are said to be weakly compatible if they commute at the coincidence point, i.e., the pair $\{\mathrm{f}, \mathrm{g}\}$ is weakly compatible pair if and only if $\mathrm{f}(\xi \mathrm{x})=\mathrm{g}(\xi \mathrm{x})$ implies that $\mathrm{fg}(\xi \mathrm{x})=\mathrm{gf}(\xi \mathrm{x})$.

Lemma2.1.22: Let $\left\{\xi \mathrm{p}_{\mathrm{n}}\right\}$ be a sequence in Menger space $(\mathrm{X}, \mathrm{F}, \Omega, \Delta)$ where $\Delta$ is continuous and $\Delta(\mathrm{x}, \mathrm{x}) \geq \mathrm{x}$ for all $\mathrm{x} \in[0,1]$. If there exists a constant $\mathrm{k} \in(0,1)$ such that $\mathrm{x}>0$ and $\mathrm{n} \in \mathbf{N} \quad \mathrm{F}_{\xi \xi p_{n}, \mathrm{p}_{\mathrm{n}+1}}(\mathrm{kx}) \geq$ $\mathrm{F}_{\xi \mathrm{p}_{\mathrm{n}-1}, \xi \mathrm{p}_{\mathrm{n}}}(\mathrm{x})$, then $\left\{\xi \mathrm{p}_{\mathrm{n}}\right\}$ is a Cauchy sequence.

Lemma 2.1.23: If $(X, d, \Omega)$ is a random metric space, then the metric $d$ induces a mapping $F: X \times X \rightarrow L$, defined by $F(\xi p, \xi q)=H(x-d(\xi p, \xi q))$, $p, q \in X$ and $x \in R$. Further more if $\Delta:[0,1] \times[0,1] \rightarrow[0,1]$ is defined by $\Delta(\mathrm{a}, \mathrm{b})=\min (\mathrm{a}, \mathrm{b})$, then $(\mathrm{X}, \mathrm{F}, \Omega, \Delta)$ is a Menger space. It is complete if $(\mathrm{X}, \mathrm{d}, \Omega)$ is complete. The space $(\mathrm{X}, \mathrm{F}, \Omega, \Delta)$ so obtained is called the induced Menger space.

Lemma 2.1.24: Let $(X, F, \Omega, \Delta)$ be a random menger space. If there exists a constant $k \in(0,1)$ such that $\mathrm{F}_{\xi x, \xi y}(k t) \geq \mathrm{F}_{\xi x, \xi y}(t)$, for all $\xi \mathrm{x}, \xi \mathrm{y} \in \mathrm{X}$ and $\mathrm{t}>0$ then $\mathrm{x}=\mathrm{y}$.

\subsection{COMMON FIXED POIONT THEOREMS IN RANDOM PROBABILISTIC METRIC SPACES}

Theorem 2.2.1: Let $(\mathrm{X}, \mathrm{F}, \Omega, \Delta)$ be a complete random menger space where $\Delta$ is continuous and $\Delta(\mathrm{t}, \mathrm{t}) \geq \mathrm{t}$ for all $\mathrm{t} \in[0,1]$. Let $\mathrm{A}, \mathrm{B}, \mathrm{T}$ and $\mathrm{S}$ be mappings from $\mathrm{X}$ into itself such that

2.2.1.(a) $S(X) \subset A(X)$ and $T(X) \subset B(X)$

2.2.1.2. $\mathrm{AB}=\mathrm{BA}, \mathrm{ST}=\mathrm{TS}$ weakly commuting

2.2.1.3. The pair $(S, A)$ and $(T, B)$ are weakly compatible

2.2.1.4. There exists a number $\mathrm{k} \in(0,1)$ such that

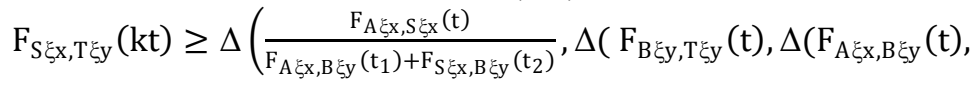




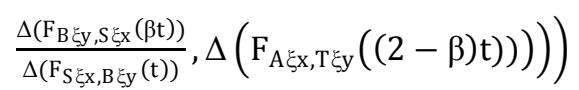

in X.

for all $\xi \mathrm{x}, \xi \mathrm{y} \in \mathrm{X}, \beta \in(0,2)$ and $\mathrm{t}>0 ; \mathrm{t}_{1}+\mathrm{t}_{2}=\mathrm{t}$. Then, $\mathrm{A}, \mathrm{B}, \mathrm{S}$ and $\mathrm{T}$ have a unique common fixed point

Proof: Since $S(X) \subset A(X)$ for any $\xi x_{0} \in X$ there exists a point $\xi x_{1} \in X$ such that $S \xi x_{0}=A \xi x_{1}$. Since $T(X) \subset$ $\mathrm{B}(\mathrm{X})$ for this point $\xi \mathrm{x}_{1}$ we can choose a point $\xi \mathrm{x}_{2} \in \mathrm{X}$ such that $\mathrm{T} \xi \mathrm{x}_{1}=\mathrm{B} \xi \mathrm{x}_{2}$.

Inductively we can find a sequence $\left\{\xi \mathrm{y}_{\mathrm{n}}\right\}$ as follows $\xi \mathrm{y}_{2 \mathrm{n}}=\mathrm{S} \xi \mathrm{x}_{2 \mathrm{n}}=\mathrm{A} \xi \mathrm{x}_{2 \mathrm{n}+1}$ and $\xi \mathrm{y}_{2 \mathrm{n}+1}=\mathrm{T} \xi \mathrm{x}_{2 \mathrm{n}+1}=$ $\mathrm{B} \xi \mathrm{x}_{2 \mathrm{n}+2}$

For $\mathrm{n}=0,1,2,3 \ldots \ldots \ldots$ for all $\mathrm{t}>0$ and $\beta=1-\mathrm{q}$ with $\mathrm{q} \in(0,1)$, we have

$$
\begin{aligned}
& \mathrm{F}_{\xi \mathrm{y}_{2 \mathrm{n}+1}, \xi \mathrm{y}_{2 \mathrm{n}+2}}(\mathrm{kt})=\mathrm{F}_{\mathrm{S} \xi \mathrm{x}_{2 \mathrm{n}+1}, T \xi \mathrm{x}_{2 \mathrm{n}+2}}(\mathrm{kt})
\end{aligned}
$$

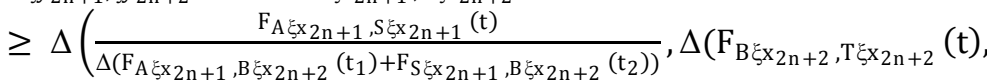

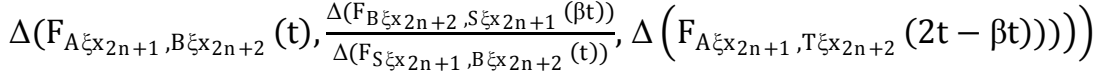

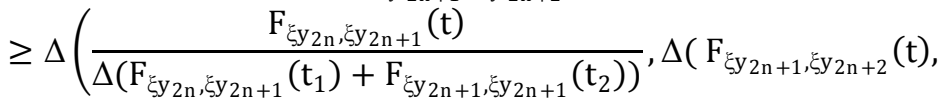

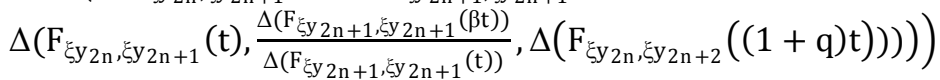

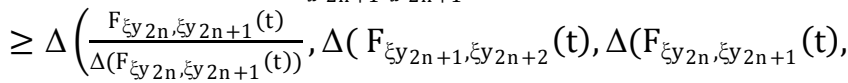

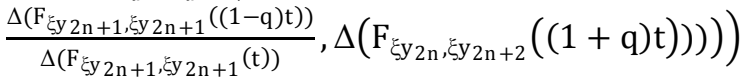

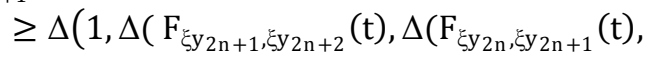

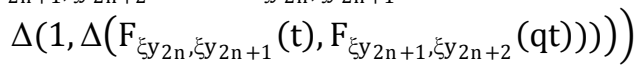

$$
\begin{aligned}
& \geq \Delta\left(\mathrm{F}_{\xi y_{2 n}, \xi y_{2 n+1}}(\mathrm{t}), \mathrm{F}_{\xi y_{2 n+1}, \xi y_{2 n+2}}(\mathrm{qt})\right) \\
& \mathrm{F}_{\xi y_{2 n+1}, \xi y_{2 n+2}}(k t) \geq \Delta\left(F_{\xi y_{2 n}, \xi y_{2 n+1}}(t), F_{\xi_{2} n+1}, \xi_{2 n+2}(q t)\right)
\end{aligned}
$$

Since $\Delta$ is continuous and the distribution function is left continuous, making $\mathrm{q} \rightarrow 1$ we have

$$
\begin{aligned}
& F_{\xi y 2 n+1, \xi y 2 n+2}(k t) \geq \Delta\left(F_{\xi y 2 n}, \xi_{y 2 n+1}(t), F_{\xi y 2 n+1}, \xi_{y 2 n+2}(t)\right) \\
& \text { Similarly } \\
& \mathrm{F}_{\xi y_{2 n+2}, \xi y_{2 n+3}}(k t) \geq \Delta\left(\mathrm{F}_{\xi y 2 n+1, \xi y_{2 n+2}}(t), F_{\xi y 2 n+2, \xi y_{2 n+3}}(t)\right)
\end{aligned}
$$

Therefore

$\mathrm{F}_{\xi y_{n}, \xi y_{n}+1}(k t) \geq \Delta\left(F_{\xi y_{n}-1, \xi y_{n}}(t), F_{\xi y_{n}, \xi y_{n}+1}(t)\right)$ for all $n \in N$

Consequently

$F_{\xi y_{n}, \xi y_{n}+1}(t) \geq \Delta\left(F_{\xi y_{n}-1, \xi y_{n}}\left(k^{-1} t\right), F_{\xi y_{n}, \xi y_{n}+1}\left(k^{-1} t\right)\right)$ for all $n \in N$

Repeated application of this inequality will imply that

$$
\begin{aligned}
& \qquad \mathrm{F}_{\xi y_{n}, \xi y_{n}+1}(t) \geq \Delta\left(F_{\xi y_{n}-1, \xi y_{n}}\left(k^{-1} t\right), F_{\xi y_{n}, \xi y_{n}+1}\left(k^{-1} t\right)\right) \geq \cdots \ldots \ldots \\
& \geq \Delta\left(F_{\xi y_{n}-1, \xi y_{n}}\left(k^{-1} t\right), F_{\xi y_{n}, \xi y_{n}+1}\left(k^{-i} t\right)\right), i \in N \\
& \text { Since } F_{\xi y_{n}, \xi y_{n}+1}\left(k^{-i} t\right) \rightarrow 1 \text { as } i \rightarrow \infty \text {, it follows that } \\
& F_{\xi y_{n}, \xi y_{n}+1}(t) \geq \Delta\left(F_{\xi y_{n-1}, \xi y_{n}}\left(k^{-1} t\right)\right) \text { for all } n \in N \\
& \text { Consequently } \\
& F_{\xi y_{n}, \xi y_{n}+1}(k t) \geq \Delta\left(F_{\xi y_{n}-1, \xi y_{n}}(t)\right) \text { for all } n \in N
\end{aligned}
$$

Therefore $\left\{\xi_{\mathrm{n}}\right\}$ is a Cauchy sequence in $\mathrm{X}$.

Since $\mathrm{X}$ is complete, $\left\{\xi \mathrm{y}_{\mathrm{n}}\right\}$ converges to a point $\mathrm{z} \in \mathrm{X}$.

Since $\left\{S \xi x_{2 n}\right\},\left\{T \xi x_{2 n+1}\right\},\left\{A \xi x_{2 n+1}\right\}$ and $\left\{B \xi x_{2 n+2}\right\}$ are subsequences of $\left\{\xi y_{n}\right\}$, they also converge to the point $\xi \mathrm{z}$,

$$
\text { i. e. as } n \rightarrow \infty, S \xi x_{2 n}, T \xi x_{2 n+1}, A \xi x_{2 n+1} B \xi x_{2 n+2} \rightarrow \xi z \text {. }
$$

SinceS $(X) \subset A(X)$, there exists a point $\mathrm{u} \in \mathrm{X}$ such that $\mathrm{Au}=\mathrm{z}$.

By putting $\mathrm{x}=\xi \mathrm{u}$ and $\mathrm{y}=2 \mathrm{n}-1$ with $\beta=1$

we have,

$\mathrm{F}_{\mathrm{S} \xi \mathrm{u}, \mathrm{T} \xi \mathrm{x}_{2 \mathrm{n}-1}}(\mathrm{kt})$ 


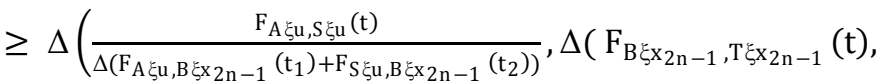

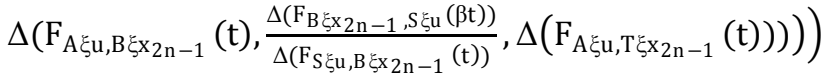

Proceeding limit as $\mathrm{n} \rightarrow \infty$, we have

$\mathrm{F}_{\mathrm{S} \xi u, \xi z}(\mathrm{kt})$

Consequently

$$
\begin{gathered}
\geq \Delta\left(\frac{\mathrm{F}_{\mathrm{z}, \mathrm{Su}}(\mathrm{t})}{\Delta\left(\mathrm{F}_{\xi \mathrm{z}, \xi \mathrm{z}}\left(\mathrm{t}_{1}\right)+\mathrm{F}_{\mathrm{S} \xi \mathrm{u}, \xi \mathrm{z}}\left(\mathrm{t}_{2}\right)\right)}, \Delta\left(\mathrm{F}_{\xi \mathrm{z}, \xi \mathrm{z}}(\mathrm{t}), \Delta\left(\mathrm{F}_{\xi \mathrm{z}, \xi \mathrm{z}}(\mathrm{t}), \frac{\Delta\left(\mathrm{F}_{\xi \mathrm{z}, S \xi \mathrm{u}}(\mathrm{t})\right)}{\Delta\left(\mathrm{F}_{\mathrm{S} \xi \mathrm{u}, \xi \mathrm{z}}(\mathrm{t})\right)}, \Delta\left(\mathrm{F}_{\xi \mathrm{z}, \xi \mathrm{z}}(\mathrm{t})\right)\right)\right)\right) \\
\geq \Delta\left(\frac{\mathrm{F}_{\xi \mathrm{z}, \mathrm{S} \xi \mathrm{u}}(\mathrm{t})}{\Delta\left(\mathrm{F}_{\xi \mathrm{z}, \mathrm{S} \xi \mathrm{u}}(\mathrm{t})\right)}, \Delta\left(\mathrm{F}_{\xi \mathrm{z}, \xi \mathrm{z}}(\mathrm{t}), \Delta\left(\mathrm{F}_{\xi \mathrm{z}, \xi \mathrm{z}}(\mathrm{t}), \frac{\Delta\left(\mathrm{F}_{\xi \mathrm{z}, \mathrm{S} \xi \mathrm{u}}(\mathrm{t})\right)}{\Delta\left(\mathrm{F}_{\mathrm{S} \xi \mathrm{u}, \xi \mathrm{z}}(\mathrm{t})\right)}, \Delta\left(\mathrm{F}_{\xi \mathrm{z}, \xi \mathrm{z}}(\mathrm{t})\right)\right)\right)\right) \\
\geq \Delta\left(\mathrm{F}_{\xi \mathrm{z}, \mathrm{S} \xi \mathrm{u}}(\mathrm{t}), \Delta(1, \Delta(1,1, \Delta(1)))\right)
\end{gathered}
$$

which tends to 1 and $\mathrm{j}$ tends to $\infty(\mathrm{j} \in \mathrm{N})$

$$
\mathrm{F}_{\mathrm{S} \xi \mathrm{u}, \xi \mathrm{z}}(\mathrm{t}) \geq \mathrm{F}_{\mathrm{S} \xi \mathrm{u}, \xi \mathrm{z}}\left(\mathrm{k}^{-1} \mathrm{t}\right) \geq \cdots \ldots \ldots \ldots \mathrm{F}_{\mathrm{S} \xi \mathrm{u}, \xi \mathrm{z}}\left(\mathrm{k}^{-\mathrm{j}} \mathrm{t}\right)
$$

Therefore $\mathrm{S} \xi \mathrm{u}=\xi \mathrm{z}$ and thus $\mathrm{A} \xi \mathrm{u}=\mathrm{S} \xi \mathrm{u}=\xi \mathrm{z}$.

Since $\mathrm{T}(\mathrm{X}) \subset \mathrm{B}(\mathrm{X})$, there exists a point $\xi \mathrm{v} \in \mathrm{X}$ such that $\mathrm{B} \xi \mathrm{v}=\xi \mathrm{z}$.

Then by putting $\xi \mathrm{x}=\xi \mathrm{u}$ and $\xi \mathrm{y}=\xi \mathrm{v}$ with $\beta=1$ in we have

$$
\begin{aligned}
& \mathrm{F}_{\mathrm{S} \xi \mathrm{u}, \mathrm{T} \xi \mathrm{v}}(\mathrm{kt}) \\
& \geq \Delta\left(\frac{\mathrm{F}_{\mathrm{A} \xi \tilde{\mathrm{u}}, \mathrm{S} \xi \mathrm{u}}(\mathrm{t})}{\Delta\left(\mathrm{F}_{\mathrm{A} \xi \mathrm{u}, \mathrm{B} \xi \mathrm{v}}\left(\mathrm{t}_{1}\right)+\mathrm{F}_{\mathrm{S} \xi \mathrm{u}, \mathrm{B} \xi \mathrm{v}}\left(\mathrm{t}_{2}\right)\right)}, \Delta\left(\mathrm{F}_{\mathrm{B} \xi \mathrm{v}, \mathrm{T} \xi \mathrm{v}}(\mathrm{t}),\right.\right. \\
& \left.\left.\Delta\left(\mathrm{F}_{\mathrm{A} \xi \mathrm{u}, \mathrm{B} \xi \mathrm{v}}(\mathrm{t}), \frac{\Delta\left(\mathrm{F}_{\mathrm{B} \xi \mathrm{v}, \mathrm{S} \xi \mathrm{u}}(\beta \mathrm{t})\right)}{\Delta\left(\mathrm{F}_{\mathrm{S} \xi \mathrm{u}, \mathrm{B} \xi \mathrm{v}}(\mathrm{t})\right)}, \Delta\left(\mathrm{F}_{\mathrm{A} \xi \mathrm{u}, \mathrm{T} \xi \mathrm{v}}(\mathrm{t})\right)\right)\right)\right) \\
& \geq \Delta\left(\frac{\mathrm{F}_{\mathrm{A} \xi \mathrm{u}, \mathrm{S} \xi \mathrm{u}}(\mathrm{t})}{\Delta\left(\mathrm{F}_{\mathrm{A} \xi \xi \mathrm{u}, \mathrm{S} \xi \mathrm{u}}(\mathrm{t})\right)}, \Delta\left(\mathrm{F}_{\mathrm{B} \xi \mathrm{v}, \mathrm{T} \xi \mathrm{v}}(\mathrm{t}), \Delta\left(\mathrm{F}_{\mathrm{A} \xi \mathrm{u}, \mathrm{B} \xi \mathrm{v}}(\mathrm{t}), \frac{\Delta\left(\mathrm{F}_{\mathrm{B} \xi \mathrm{v}, \mathrm{S} \xi \mathrm{u}}(\beta \mathrm{t})\right)}{\Delta\left(\mathrm{F}_{\mathrm{S} \xi \mathrm{u}, \mathrm{B} \xi \mathrm{v}}(\mathrm{t})\right)}, \Delta\left(\mathrm{F}_{\mathrm{A} \xi \mathrm{u}, \mathrm{T} \xi \mathrm{v}}(\mathrm{t})\right)\right)\right)\right)
\end{aligned}
$$

Using above we have we have

$$
\begin{aligned}
\mathrm{F}_{\mathrm{S} \xi \mathrm{u}, T \xi \mathrm{v}}(\mathrm{kt}) & \geq \Delta\left(\frac{\mathrm{F}_{\xi \mathrm{z}, \xi_{\mathrm{z}}}(\mathrm{t})}{\Delta\left(\mathrm{F}_{\xi \mathrm{z}, \xi_{\mathrm{z}}}(\mathrm{t})\right)}, \Delta\left(\mathrm{F}_{\xi \mathrm{z}, \mathrm{T} \xi \mathrm{v}}(\mathrm{t}), \Delta\left(\mathrm{F}_{\xi \mathrm{z}, \xi \mathrm{z}}(\mathrm{t}), \frac{\Delta\left(\mathrm{F}_{\xi z}, \xi_{\mathrm{z}}(\mathrm{t})\right)}{\Delta\left(\mathrm{F}_{\xi z}, \xi_{\mathrm{z}}(\mathrm{t})\right)}, \Delta\left(\mathrm{F}_{\xi \mathrm{z}, \mathrm{T} \xi \mathrm{v}}(\mathrm{t})\right)\right)\right)\right) \\
\mathrm{F}_{\xi \mathrm{z}, \mathrm{T} \xi \mathrm{v}}(\mathrm{kt}) & \geq \Delta\left(\mathrm{F}_{\xi \mathrm{z}, \mathrm{T} \xi \mathrm{v}}(\mathrm{t})\right)
\end{aligned}
$$

As above we have $\mathrm{T} \xi \mathrm{v}=\xi \mathrm{z}$.

Therefore $\mathrm{A} \xi \mathrm{u}=\mathrm{S} \xi \mathrm{u}=\mathrm{T} \xi \mathrm{v}=\mathrm{B} \xi \mathrm{v}=\xi \mathrm{z}$.

Since pair of maps $S$ and $A$ are weakly compatible, then $S u=A u$ implies $S(A) \xi u=(A) S \xi u$, i.e. $\mathrm{S} \xi \mathrm{z}=\mathrm{A} \xi \mathrm{z}$.

Now to show that $\mathrm{z}$ is a fixed point of $\mathrm{P}$ so by putting $\xi \mathrm{x}=\xi \mathrm{z}$ and $\xi \mathrm{y}=\xi_{\mathrm{x}}$ n with $\quad \beta=1$

$$
\begin{aligned}
& \mathrm{F}_{\mathrm{S} \xi \mathrm{z}, \mathrm{T} \xi \mathrm{x}_{2 \mathrm{n}}}(\mathrm{kt}) \\
& \geq \Delta\left(\frac{\mathrm{F}_{\mathrm{A} \xi \mathrm{z}, \mathrm{S} \xi \mathrm{z}}(\mathrm{t})}{\Delta\left(\mathrm{F}_{\mathrm{A} \xi z, \mathrm{~B} \xi \mathrm{x}_{2 n} \mathrm{n}}\left(\mathrm{t}_{1}\right)+\mathrm{F}_{\mathrm{S} \xi \mathrm{z}, \mathrm{B} \xi \mathrm{x}_{2 \mathrm{n}}}\left(\mathrm{t}_{2}\right)\right)}, \Delta\left(\mathrm{F}_{\mathrm{B} \xi \mathrm{x}_{2 \mathrm{n}}, \mathrm{T} \xi \mathrm{x}_{2 \mathrm{n}}}(\mathrm{t}),\right.\right. \\
& \left.\left.\Delta\left(\mathrm{F}_{\mathrm{A} \xi \mathrm{z}, \mathrm{B} \xi \mathrm{x}_{2 n}}(\mathrm{t}), \frac{\Delta\left(\mathrm{F}_{\mathrm{B} \xi \mathrm{x}_{2 n}, \mathrm{~S} \xi \mathrm{z}}(\mathrm{t})\right)}{\Delta\left(\mathrm{F}_{\mathrm{S} \xi \mathrm{z}, \mathrm{B} \xi \mathrm{x}_{2 n} \mathrm{n}}(\mathrm{t})\right)}, \Delta\left(\mathrm{F}_{\mathrm{A} \xi \mathrm{z}, \mathrm{T} \xi \mathrm{x}_{2 n}}(\mathrm{t})\right)\right)\right)\right)
\end{aligned}
$$

Using above, we have

$$
\begin{aligned}
& \mathrm{F}_{\mathrm{S} \xi \mathrm{z}, \xi \mathrm{z}}(\mathrm{kt})
\end{aligned}
$$

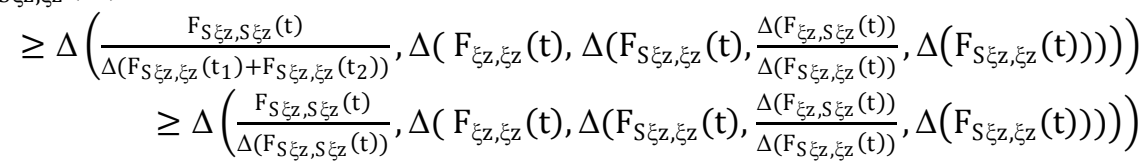

Thus we have $S \xi z=\xi z$. Hence $S \xi z=\xi z=A \xi z$.

Similarly, pair of maps $\mathrm{T}$ and $\mathrm{B}$ is weakly compatible, then $\mathrm{T} \xi \mathrm{u}=\mathrm{B} \xi \mathrm{u}$.

Now we show that $\mathrm{z}$ is a fixed point of $\mathrm{T}$ so by putting $\xi \mathrm{x}=\xi \mathrm{x}_{2 \mathrm{n}}$ and $\xi \mathrm{y}=\xi \mathrm{z}$ with $\beta=1$

$$
\begin{aligned}
& \mathrm{F}_{\mathrm{S} \xi \mathrm{x}_{2 \mathrm{n}}, T \xi \mathrm{z}}(\mathrm{kt}) \geq \Delta\left(\frac{\mathrm{F}_{\mathrm{A} \xi \mathrm{x}_{2 n},}, \mathrm{~S} \xi \mathrm{x}_{2 \mathrm{n}}(\mathrm{t})}{\Delta\left(\mathrm{F}_{\mathrm{A} \xi \mathrm{x}_{2 n} \mathrm{n}, \mathrm{B} \xi \mathrm{z}}\left(\mathrm{t}_{1}\right)+\mathrm{F}_{\mathrm{S} \xi \mathrm{x}_{2 n}, \mathrm{~B} \xi \mathrm{z}}\left(\mathrm{t}_{2}\right)\right)}, \Delta\left(\mathrm{F}_{\mathrm{B} \xi \mathrm{z}, \mathrm{T} \xi \mathrm{z}}(\mathrm{t}),\right.\right. \\
& \left.\left.\Delta\left(\mathrm{F}_{\mathrm{A} \xi \mathrm{x}_{2 \mathrm{n}}, \mathrm{B} \xi \mathrm{z}}(\mathrm{t}), \frac{\Delta\left(\mathrm{F}_{\mathrm{B}} \mathrm{z}_{\left.\mathrm{z}, S \xi \mathrm{x}_{2 \mathrm{n}}(\mathrm{Bt})\right)}\right.}{\Delta\left(\mathrm{F}_{\mathrm{S} \xi \mathrm{x}_{2 n}, \mathrm{~B} \xi \mathrm{z}}(\mathrm{t})\right)}, \Delta\left(\mathrm{F}_{\mathrm{A} \xi \mathrm{x}_{2 \mathrm{n}}, \mathrm{T} \xi \mathrm{z}}(\mathrm{t})\right)\right)\right)\right) \\
& \geq \Delta\left(\frac{\mathrm{F}_{\mathrm{A} \xi \mathrm{x}_{2 n}, \mathrm{~S} \xi \mathrm{x}_{2 \mathrm{n}}}(\mathrm{t})}{\Delta\left(\mathrm{F}_{\mathrm{A} \xi \mathrm{x}_{2 n}, S \xi \mathrm{x}_{2 \mathrm{n}}}(\mathrm{t})\right)}, \Delta\left(\mathrm{F}_{\mathrm{B} \xi \mathrm{z}, \mathrm{T} \xi \mathrm{z}}(\mathrm{t}), \Delta\left(\mathrm{F}_{\mathrm{A} \xi \mathrm{x}_{2 \mathrm{n}}, \mathrm{B} \xi \mathrm{z}}(\mathrm{t}),\right.\right.\right. \\
& \left.\left.\left.\frac{\Delta\left(\mathrm{F}_{\mathrm{B} \xi \bar{z}, \mathrm{~S} \xi \mathrm{x}_{2 \mathrm{n}}}(\mathrm{\beta t})\right)}{\Delta\left(\mathrm{F}_{\mathrm{S} \xi \mathrm{x}_{2 n}, \mathrm{~B} \xi \mathrm{z}}(\mathrm{t})\right)}, \Delta\left(\mathrm{F}_{\mathrm{A} \xi \mathrm{x}_{2 \mathrm{n}}, \mathrm{T} \xi \mathrm{z}}(\mathrm{t})\right)\right)\right)\right) \\
& \text { Proceeding limit as } \mathrm{n} \rightarrow \infty \text {, we have }
\end{aligned}
$$




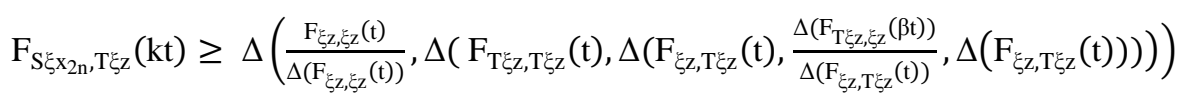

Thus we haveT $\xi_{\mathrm{z}}=\xi_{\mathrm{z}}$. Hence $\mathrm{T} \xi_{\mathrm{z}}=\xi_{\mathrm{z}}=\mathrm{B} \xi_{\mathrm{z}}$.

By combining the above results, we have

$\mathrm{S} \xi_{\mathrm{Z}}=\mathrm{A} \xi_{\mathrm{Z}}=\mathrm{T} \xi_{\mathrm{Z}}=\mathrm{B} \xi_{\mathrm{Z}}=\xi_{\mathrm{z}}$.

That is $\mathrm{z}$ is a common fixed point of $\mathrm{S}, \mathrm{T}, \mathrm{A}$ and $\mathrm{B}$.

For uniqueness, let $\xi_{\mathrm{W}}\left(\xi_{\mathrm{W}} \neq \xi_{\mathrm{Z}}\right)$ be another common fixed point of $\mathrm{S}, \mathrm{T}, \mathrm{A}$ and $\mathrm{B}$ and $\beta=1$, then we write

$$
\begin{aligned}
& \mathrm{F}_{\mathrm{S} \xi \mathrm{z}, \mathrm{T} \xi \mathrm{w}}(\mathrm{kt})
\end{aligned}
$$

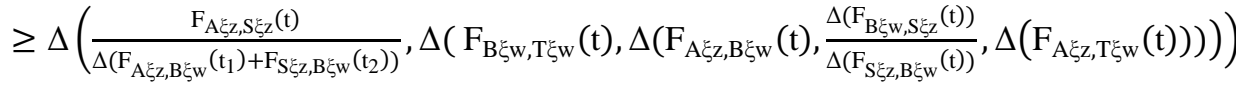

$$
\begin{aligned}
& \mathrm{F}_{\mathrm{S} \xi \mathrm{Z}, \mathrm{T} \xi \mathrm{w}}(\mathrm{kt})
\end{aligned}
$$

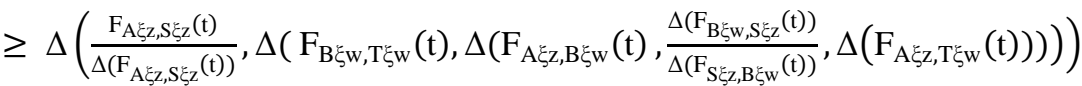

It follows that

$$
\begin{aligned}
& \quad \mathrm{F}_{\xi \mathrm{z}, \xi \mathrm{w}}(\mathrm{kt}) \geq \Delta\left(\frac{\mathrm{F}_{\xi z, \xi \mathrm{z}}(\mathrm{t})}{\Delta\left(\mathrm{F}_{\xi \mathrm{z}, \xi \mathrm{z}}(\mathrm{t})\right)}, \Delta\left(\mathrm{F}_{\xi \mathrm{w}, \xi \mathrm{w}}(\mathrm{t}), \Delta\left(\mathrm{F}_{\xi \mathrm{z}, \xi \mathrm{w}}(\mathrm{t}), \frac{\Delta\left(\mathrm{F}_{\xi \mathrm{w}, \xi \mathrm{z}}(\beta \mathrm{t})\right)}{\Delta\left(\mathrm{F}_{\xi z, \xi \mathrm{w}}(\mathrm{t})\right)}, \Delta\left(\mathrm{F}_{\xi \mathrm{z}, \xi \mathrm{w}}(\mathrm{t})\right)\right)\right)\right) \\
& \geq \Delta\left(1, \Delta\left(1, \Delta\left(\mathrm{F}_{\mathrm{z}, \mathrm{w}}(\mathrm{t}), 1, \Delta\left(\mathrm{F}_{\mathrm{z}, \mathrm{w}}(\mathrm{t})\right)\right)\right)\right) \\
& \quad \geq \mathrm{F}_{\xi z, \xi \mathrm{w}}(\mathrm{t})
\end{aligned}
$$

Thus we have $\xi_{\mathrm{z}}=\xi \mathrm{w}$. This completes the proof of the theorem.

COROLLARY 3.1: Let $(\mathrm{X}, \mathrm{F}, \Omega, \Delta)$ be a complete random menger space where $\Delta$ is continuous and $\Delta(\mathrm{t}, \mathrm{t}) \geq$ $\mathrm{t}$ for all $\mathrm{t} \in[0,1]$. Let $\mathrm{T}$ and $\mathrm{S}$ be mappings from $\mathrm{X}$ into itself such that

3.1.1. $\mathrm{ST}=\mathrm{TS}$ weakly commuting

3.1.2. There exists a number $\mathrm{k} \in(0,1)$ such that

$$
\mathrm{F}_{\text {Sx,Ty }}(\mathrm{kt}) \geq \Delta\left(\frac{\mathrm{F}_{\mathrm{x}, \mathrm{Sx}}(\mathrm{t})}{\mathrm{F}_{\mathrm{x}, \mathrm{y}}\left(\mathrm{t}_{1}\right)+\mathrm{F}_{\mathrm{Sx}, \mathrm{y}}\left(\mathrm{t}_{2}\right)}, \Delta\left(\mathrm{F}_{\mathrm{y}, \mathrm{Ty}}(\mathrm{t}), \Delta\left(\mathrm{F}_{\mathrm{x}, \mathrm{y}}(\mathrm{t}), \frac{\Delta\left(\mathrm{F}_{\mathrm{y}, \mathrm{Sx}}(\beta \mathrm{t})\right)}{\Delta\left(\mathrm{F}_{\mathrm{Sx}, \mathrm{y}}(\mathrm{t})\right)}, \Delta \mathrm{F}_{\mathrm{x}, \mathrm{Ty}}((2-\beta) \mathrm{t})\right)\right)\right)
$$

for all $\mathrm{x}, \mathrm{y} \in \mathrm{X}, \beta \in(0,2)$ and $\mathrm{t}>0 ; \mathrm{t}_{1}+\mathrm{t}_{2}=\mathrm{t}$

Then $\mathrm{S}$ and $\mathrm{T}$ have a unique common fixed point in $\mathrm{X}$.

Proof: Put $\mathrm{A}=\mathrm{B}=\mathrm{I}$ in the proof of theorem

COROLLARY 3.2: Let $(X, F, \Omega, \Delta)$ be a complete Menger space where $\Delta$ is continuous and $\Delta(t, t) \geq$ $\mathrm{t}$ for all $\mathrm{t} \in[0,1]$. Let $\mathrm{A}, \mathrm{B}, \mathrm{T}$ and $\mathrm{S}$ be mappings from $\mathrm{X}$ into itself such that

3.2.1. $S(X) \subset B(X)$

3.2.2. The pair $(S, B)$ is weakly compatible

3.2.3. There exists a number $\mathrm{k} \in(0,1)$ such that

$\mathrm{F}_{\mathrm{Sx}, \mathrm{Sy}}(\mathrm{kt})$

$\left.\geq \Delta\left(\frac{\mathrm{F}_{\mathrm{Bx}, \mathrm{Sx}}(\mathrm{t})}{\mathrm{F}_{\mathrm{Bx}, \mathrm{By}}\left(\mathrm{t}_{1}\right)+\mathrm{F}_{\mathrm{Sx}, \mathrm{By}}\left(\mathrm{t}_{2}\right)}, \Delta\left(\mathrm{F}_{\mathrm{By}, \mathrm{Sy}}(\mathrm{t}), \Delta\left(\mathrm{F}_{\mathrm{Bx}, \mathrm{By}}(\mathrm{t}), \frac{\Delta\left(\mathrm{F}_{\mathrm{By}, \mathrm{Sx}}(\beta \mathrm{t})\right)}{\Delta\left(\mathrm{F}_{\mathrm{Sx}, \mathrm{By}}(\mathrm{t})\right)}, \Delta \mathrm{F}_{\mathrm{Bx}, \mathrm{Sy}}((2-\beta) \mathrm{t})\right)\right)\right)\right)$

for all $\mathrm{x}, \mathrm{y} \in \mathrm{X}, \beta \in(0,2)$ and $\mathrm{t}>0 ; \mathrm{t}_{1}+\mathrm{t}_{2}=\mathrm{t}$.

Then $\mathrm{S}$ and $\mathrm{B}$ have a unique common fixed point in $\mathrm{X}$.

Proof: Put $\mathrm{T}=\mathrm{S}$ and $\mathrm{A}=\mathrm{B}$ in the proof of above theorem

Theorem 4.1 Let A, B, P and Q are self maps on a complete random probabilistic metric space $(X, F, \Omega)$ satisfying:

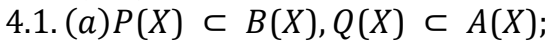

$4.1(b) F_{P \xi x, Q \xi y}(k t) \geq \max \left\{F_{A \xi x, B \xi y}(t), \frac{F_{P \xi x, A \xi x}(t)+F_{Q \xi x, B \xi x}(k t)}{2}\right\}$;

for all $\xi \mathrm{x}, \xi \mathrm{y} \in \mathrm{X}, \mathrm{t}>0, \mathrm{k} \in(0,1)$

4.1(c) If one of $P(X), B(X), Q(X), A(X)$ is complete subset of $\mathrm{X}$ then

(i) $\mathrm{P}$ and $\mathrm{A}$ have a coincidence point and

(ii) Q and B have a coincidence point and

if the pair $(\mathrm{P}, \mathrm{A})$ and $(\mathrm{Q}, \mathrm{B})$ are weakly compatible, then $\mathrm{A}, \mathrm{B}, \mathrm{P}$ and $\mathrm{Q}$ have a unique common fixed point in X.

Proof: Since $P(X) \subset B(X)$ and $Q(X) \subset A(X)$ so we can define sequences

$\left(\xi x_{n}\right)$ and $\left(\xi y_{n}\right)$ in $X$ such that for all $\mathrm{n}=0,1,2,3, \ldots$

$$
\xi y_{2 n+1}=P \xi x_{2 n}=B \xi x_{2 n+1}, \xi y_{2 n+2}=Q \xi x_{2 n+1}=A \xi x_{2 n+2}
$$


Now we have,

Similarly,

$$
\begin{aligned}
& F_{P \xi x_{2 n}, Q \xi x_{2 n+1}}(k t) \geq \max \left\{F_{A \xi x_{2 n}, B \xi x_{2 n+1}}(t), \frac{F_{P \xi x_{2 n}, A \xi x_{2 n}}(t)+F_{Q \xi x_{2 n}, B \xi x_{2 n}}(k t)}{2}\right\} \\
& F_{\xi y_{2 n+1}, \xi y_{2 n+2}}(k t) \geq \max \left\{F_{\xi y_{2 n}, \xi y_{2 n+1}}(t), \frac{F_{\xi y_{2 n+1}, \xi y_{2 n}}(t)+F_{\xi y}}{2}(k t) \geq F_{\xi y_{2 n+1}, \xi \xi y_{2 n}(k t)}\right\} \\
& F_{\xi y_{2 n+1}, \xi}(t)
\end{aligned}
$$

In general for any $\mathrm{n}$ and $\mathrm{t}$,

$$
F_{\xi y_{2 n+2}, \xi y_{2 n+3}}(k t) \geq F_{\xi y_{2 n+1}, \xi y_{2 n+2}}(t) .
$$

we have

$$
F_{\xi y_{n}, \xi y_{n+1}}(k t) \geq F_{\xi y_{n-1}, \xi y_{n}}(t) .
$$

Hence $\xi y_{n}$ is a Cauchy sequence in X. By compleness, $\xi y_{n} \rightarrow \xi z \in X$. Thus the subsequence $\left\{\xi y_{2 n}\right\},\left\{\xi y_{2 n+1}\right\}$ and $\left\{\xi y_{2 n+2}\right\}$ also converses to $\xi z$. Therefore $\left\{B \xi x_{2 n+1}\right\}$,

$\left\{P \xi x_{2 n}\right\},\left\{Q \xi x_{2 n+1}\right\}$ and $\left\{A \xi x_{2 n}\right\}$ also converses to $\xi z$. Now suppose $A(X)$ is complete. Note that the subsequence $\xi y_{2 n+2}$ contained in $\mathrm{A}(\mathrm{X})$ and has a limit in $\mathrm{A}(\mathrm{X})$ say $\xi$ z. Let $\xi w \in(A)^{-1}(\xi z)$. Then $\mathrm{A} \xi \mathrm{w}=$ $\xi \mathrm{z}$.

now consider

$$
\begin{aligned}
& F_{P_{\xi w}, Q \xi x_{2 n+1}}(k t) \geq \max \left\{F_{A_{\xi w}, B \xi x_{2 n+1}}(t), \frac{F_{P \xi_{W}, A \xi_{W}}(t)+F_{Q \zeta W}, B \xi_{W}}{}(k t)\right\}
\end{aligned}
$$

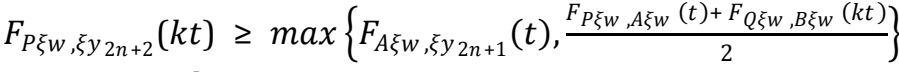

taking limit $\mathrm{n} \rightarrow \infty$, we have

$$
\begin{aligned}
& F_{P \xi W, \xi_{z}}(k t) \geq \max \left\{F_{\xi z, \xi z}(t), \frac{F_{P \xi W}, \xi_{z}(t)+F_{Q \zeta w, B \xi w}(k t)}{2}\right\} \\
& F_{P \xi w, \xi z}(k t) \geq F_{\xi z, \xi z}(t)=1 \\
& F_{P \xi_{w}, \xi_{z}}(k t)=1 \Rightarrow P \xi w=\xi z \text {. }
\end{aligned}
$$

Since $A \xi w=\xi z$ so $\xi w$ is a coincidence point of $\mathrm{P}$ and $\mathrm{A}$.

Since $P(X) \subset B(X)$ ans $P \xi w=\xi z$ implies that $\xi z \in B(X)$. Let $\xi v \in(B)^{-1} \xi z$,

then $\mathrm{B} \xi \mathrm{v}=\xi \mathrm{z}$.

now consider

$$
\begin{aligned}
& F_{P \xi x_{2 n}, Q \xi v}(k t) \geq \max \left\{F_{A \xi x_{2 n}, B \xi v}(t), \frac{F_{P \xi x_{2 n}, A \xi x_{2 n}}(t)+F_{Q \xi x_{2 n}, B \xi x_{2 n}}(k t)}{2}\right\} \\
& F_{\xi y_{2 n+1}, Q \xi v}(k t) \geq \max \left\{F_{\xi y_{2 n}, B \xi v}(t), \frac{F_{\xi y 2 n+1, \xi} y_{2 n}(t)+F_{\xi y} y_{2 n+1}, \xi y y_{2 n}}{2}\right\}
\end{aligned}
$$

Taking limit $\mathrm{n} \rightarrow \infty$, we have

$$
\begin{aligned}
& F_{\xi_{z}, Q \xi v}(k t) \geq \max \left\{F_{\xi_{z}, \xi_{z}}(t), \frac{F_{\xi_{z}, \xi_{z}}(t)+F_{\xi_{z}, \xi_{z}}(k t)}{2}\right\} \\
& F_{\xi z, Q \xi v}(k t) \geq F_{\xi z, \xi_{z}}(t)=1 \\
& F_{\xi z, Q \xi v}(k t)=1 \Rightarrow Q \xi v=\xi z
\end{aligned}
$$

Since $\mathrm{B} \xi \mathrm{v}=\xi \mathrm{z}$ so $\mathrm{v}$ is a coincidence point of $\mathrm{Q}$ and $\mathrm{B}$.

Since the pair $(\mathrm{P}, \mathrm{A})$ is weakly compatible therefore $\mathrm{P}$ and $\mathrm{A}$ commute at their coincidence point that is $P A \xi w=A P \xi w$ or $P \xi z=A \xi z$ and the pair $(\mathrm{Q}, \mathrm{B})$

is weakly compatible therefore $\mathrm{Q}$ and $\mathrm{B}$ commute at their coincidence point that is $Q B \xi v=B Q \xi v$ or $Q \xi z=B \xi z$.

Now we will prove that $P z=z$. By (b), we have

Taking limit $n \rightarrow \infty$, we have

$$
\begin{aligned}
& F_{P \xi z, Q \xi x_{2 n+1}}(k t) \geq \max \left\{F_{A \xi_{z}, B \xi x_{2 n+1}}(t), \frac{F_{P \xi_{z}, A \xi_{z}}(t)+F_{Q \xi_{z}, B \xi_{z}}(k t)}{2}\right\} \\
& F_{P \xi z, \xi y_{2 n+2}}(k t) \geq \max \left\{F_{A \xi z, \xi y_{2 n+1}}(t), \frac{F_{P \xi z, A \xi z}(t)+F_{Q \xi z, B \xi z}(k t)}{2}\right\}
\end{aligned}
$$

$$
\begin{aligned}
& F_{P \xi_{Z}, \xi_{Z}}(k t) \geq \max \left\{F_{A \xi_{z}, \xi_{Z}}(t), \frac{F_{P \xi_{z}, A \xi_{Z}}(t)+F_{Q \xi_{z}, B \xi_{Z}}(k t)}{2}\right\} \\
& F_{P \xi_{Z}, \xi_{Z}}(k t) \geq \max \left\{F_{A \xi_{z}, \xi_{Z}}(t), 1\right\}
\end{aligned}
$$

since $A \xi z=P \xi z$ and $Q \xi z=B \xi z$

$$
F_{P \xi z, \xi z}(k t)=1 \text { then } P \xi z=\xi z \text {. }
$$

Similarly we will prove that $Q \xi z=\xi z$. By (b), we have

Taking limit $n \rightarrow \infty$, we have

$$
\begin{aligned}
& F_{P \xi x_{2 n}, Q \xi_{Z}}(k t) \geq \max \left\{F_{A \xi x_{2 n}, B \xi z}(t), \frac{F_{P \xi x_{2 n}, A \xi x_{2 n}}(t)+F_{Q \xi x_{2 n}, B \xi x_{2 n}}(k t)}{2}\right\} \\
& F_{\xi y_{2 n+1}, Q \xi_{z}}(k t) \geq \max \left\{F_{\xi_{2 n}, B \xi_{Z}}(t), \frac{F_{\xi y_{2 n+1}, \xi y_{2 n}}(t)+F_{\xi y_{2 n+1}, \xi y_{2 n}}(k t)}{2}\right\}
\end{aligned}
$$




$$
\begin{aligned}
& F_{\xi z, Q \xi z}(k t) \geq \max \left\{F_{\xi z, B \xi z}(t), \frac{F_{\xi z, \xi_{z}}(t)+F_{\xi z, \xi z}(k t)}{2}\right\} \\
& F_{\xi z, Q \xi z}(k t) \geq \max \left\{F_{\xi z, B \xi z}(t), 1\right\} \\
& F_{\xi z, Q \xi z}(k t)=1 \text { then } Q \xi_{z}=\xi z .
\end{aligned}
$$

Hence $\mathrm{z}$ is a common fixed point of $\mathrm{A}, \mathrm{B}, \mathrm{P}$ and $\mathrm{Q}$.

Uniqueness Let $w$ is an another common fixed point of $A, B, P$ and $Q$.

then we have

$$
\begin{aligned}
& F_{P \xi w, Q \xi z}(k t) \geq \max \left\{F_{A \xi w, B \xi w}(t), \frac{F_{P \xi W, A \xi w}(t)+F_{Q \xi W, B \xi w}(k t)}{2}\right\} \\
& F_{\xi w, \xi z}(k t) \geq \max \left\{F_{\xi w, \xi z}(t), \frac{F_{(\xi w, \xi w)(t)}+F_{(\xi w, \xi w)(k t)}}{2}\right\} \\
& F_{\xi w, \xi z}(k t) \geq \max \left\{F_{\xi w, \xi z}(t), 1\right\} \\
& F_{\xi w, \xi z}(k t)=1 \Rightarrow \xi w=\xi z
\end{aligned}
$$

Hence $\xi \mathrm{z}$ is unique common fixed point of $\mathrm{A}, \mathrm{B}, \mathrm{P}$ and $\mathrm{Q}$.

\section{References:}

[1]. B. Schweizer and A. Sklar, Probabilistic Metric Spaces, North Holland (Amsterdam,1983).

[2]. D. Mihet, A Generalization of a contraction principle in probabilistic metric spaces, Part II, Int. J. Math. Math. Sci, 2005(2005),729-736.

[3]. G. Jungck, Compatible Mapping and Common Fixed Points, Internat. J. Math. and Math. Sci., 9 (1986), 771-773.

[4]. J.K., Kohli, Sachin Vashistha, D. Kumar, A Common Fixed Point Theorem for Six Mappings in Probabilistic Metric Spaces Satisfying Contractive type Implicit Relations, Int. J. of Math. Analysis, 4 (2010), 63-74.

[5]. 5. K. Menger, Statistical Metrics, Proc.Nat.Acad.Sci.U.S.A. 28 (1042), 535-537.

[6]. R.P. Pant, A Common Fixed Point Theorem Under a New Condition, Indian J. Pure Appl. Math. 30 (1999), 147-152.

[7]. S. Kumar and R. Chugh, common fixed point theorem using minimal commutative and reciprocal continuity condition in metric space, Sci. Math. Japan 56 (2002), 269-275.

[8]. S. Kumar, D. B. Pant, A Common Fixed Point Theorem in Probabilistic Metric Spaces Using Implicit Relation, Filomat 22:2 (2008),43-52.

[9]. S.N. Mishra, Common Fixed Point of Compatible Mappings in Probabilistic Metric Spaces, Math, Japon., 36 (1991), 283-289.

[10]. S.L. Singh and B.D. Pant, Common fixed point theorems in probabilistic metric spaces and extension to uniform spaces, Honam. Math. J. 6 (1984), 1-12.

[11]. S.L. Singh, B.D. Pant and R. Talwar, Fixed points of weakly commuting mappings on Menger spaces, Jnanabha 23 (1993), 115-122.

[12]. V. M. Sehgal, Some Fixed Point Theorem in Functional Analysis and Probability, Ph.D. Dissertation, Wayne State Univ. Michigan (1966). 